\title{
Análise de componentes principais entre características morfogênicas e estruturais em capim-marandu sob lotação contínua
}

\author{
Principal component analysis between morphogenetic and structural characteristics of Marandu \\ palisadegrass swards under continuous stocking
}

\author{
Sila Carneiro da Silva' André Fischer Sbrissia ${ }^{I I}$
}

\section{- NOTA -}

\section{RESUMO}

A análise de componentes principais é uma técnica de análise multivariada que tem sido usada com pouca frequência na pesquisa com plantas forrageiras. Dessa forma, o objetivo do presente trabalho foi verificar se as hipóteses já testadas de acordo com técnicas univariadas podem ser validadas por meio de análise de componentes principais. Assim, duas análises foram realizadas. Na primeira, mais de 80\% da variação do conjunto de dados foi explicada pelos primeiros três componentes principais, os quais relacionaram, basicamente, padrões de compensação tamanho/densidade populacional de perfilhos e discrepâncias na forma de se avaliar a forma do perfilho (relação folha: colmo ou razão área foliar: volume por perfilho). Na segunda, os três primeiros componentes principais explicaram $91,4 \%$ da variação total, relacionando-se, basicamente, com o processo de economia de recursos e alocação sazonal de assimilados para diferentes estruturas como forma de garantir sobrevivência e persistência das plantas na área. Os resultados mostram a potencialidade do uso da análise de componentes principais na interpretação de dados de pesquisa com plantas forrageiras. As conclusões obtidas são semelhantes àquelas obtidas com técnicas univariadas convencionais, com a vantagem de reduzir o número de variáveis globais em alguns poucos componentes principais.

Palavras-chave: Brachiaria brizantha, economia de recursos, estatística multivariada, pastagens.

\section{ABSTRACT}

The principal component analysis is a multivariate analysis technique that has not been frequently used in the interpretation of research data on forage plants. Thus, the aim of this study was to use data already published and interpreted according to univariate analysis and verify if their hypotheses could also be validated through the principal component analysis. Two principal components analysis were performed. For the first one, the following variables were considered: tiller population density, individual tillers mass, leaf area index, leaf area/tiller volume ratio and tiller appearance and survival rates. In the first analysis more than $80 \%$ of of the data set variation was explained by the first three main components, which, basically, showed patterns of tiller size / density compensation mechanisms and revealed discrepancies in the way of evaluating tiller shape (leaf : stem ratio or leaf area: volume ratio per tiller). In the second analysis, the first three principal components explained $91.4 \%$ of the total variation, which was related basically to the process of resources economy and seasonal allocation of assimilates for different plant structures as a mean of ensuring survival and persistence of plants. The results show the potential of using the principal component analysis in the interpretation of research data on forage plants and corroborates conclusions obtained using univariate methods, with the advantage of reducing the number of global variables

Key words: multivariate analysis techniques, Brachiaria brizantha, continuous grazing method.

Apesar de ser uma técnica relativamente antiga (JOLLIFFE, 1986), a análise de componentes principais (ACP), assim como outras técnicas de análise multivariada, não tem sido usada com frequência na interpretação de dados de pesquisas com plantas forrageiras. Na verdade, apesar de antiga, apenas recentemente, com o desenvolvimento de processadores computacionais mais rápidos, é que análises multivariadas puderam ser utilizadas

'Universidade de São Paulo (USP), Piracicaba, SP, Brasil.

"Universidade do Estado de Santa Catarina (UDESC), 88520-000, Lages, SC, Brasil. E-mail: sbrissia@cav.udesc.br. Autor para correspondência. 
rotineiramente e incorporadas às análises de dados (HAIR et al., 1998). Brevemente, a análise de componentes principais gera novas variáveis que são funções lineares das variáveis originais. Nesse sentido, a ACP maximiza a proporção da variância do conjunto de dados expresso por sucessivos componentes principais (CPs) que não são correlacionados entre si. A principal utilidade da ACP é reduzir a dimensionalidade do conjunto de dados, retendo tanta informação quanto possível num menor número de CPs. Assim, o primeiro componente principal (CP) é a combinação das variáveis que explica a maior proporção da variação total dos dados. O segundo $\mathrm{CP}$ define a maior variação seguinte e assim sucessivamente. Recentemente, a efetividade do uso de outra técnica de análise multivariada (Correlação Canônica) na interpretação de resultados de pesquisa com plantas forrageiras foi demonstrada por NAVE et al. (2009). O objetivo deste trabalho foi verificar se as hipóteses testadas de acordo com técnicas de estatística univariada por SBRISSIA \& DA SILVA (2008) e SBRISSIA et al. (2010) podem ser validadas por meio de análise de componentes principais. Detalhes da área experimental e dos métodos de coleta e avaliação dos dados podem ser encontrados nos mesmos trabalhos, os quais objetivaram detectar padrões de compensação tamanho/densidade populacional de perfilhos, dinâmica do perfilhamento e alterações sazonais em longevidade de folhas como forma de economia de recursos em pastos de capim-marandu sob lotação contínua.

Duas análises de CPs foram realizadas. $\mathrm{Na}$ primeira, objetivando identificar padrões do mecanismo de compensação tamanho/densidade populacional de perfilhos, foram consideradas as variáveis: densidade populacional de perfilhos (DPP), massa de perfilhos individuais (MP), índice de área foliar do dossel (IAF), razão área foliar/volume por perfilho (R), relação folha/ colmo (FC), taxa de aparecimento de perfilhos (TAP) e taxa de sobrevivência de perfilhos (TSP). Esse mecanismo é amplamente descrito na literatura, tanto para espécies de clima temperado (MATTTHEW et al., 1995), como de clima tropical (SBRISSIA et al., 2003; SBRISSIA \& DA SILVA, 2008). Os resultados indicaram que $84,8 \%$ da variação no conjunto de dados eram explicados por apenas três CPs. A escolha do número de CPs a ser utilizado depende do pesquisador. No entanto, JOLLIFFE (1986) sugeriu que eigenvalues menores que um poderiam ser descartados ou, ainda, dependendo do pesquisador e da natureza dos dados, poderia se escolher componentes que explicassem, por exemplo, 60\% da variação nos dados. Esse mesmo autor também sugeriu que as correlações entre componentes principais e variáveis originais inferiores a 0,25 fossem descartadas. O primeiro CP explicou 39\% da variação total dos dados e relacionou basicamente aspectos do mecanismo de compensação tamanho/densidade populacional (Tabela 1). Nesse primeiro CP, a DPP esteve positivamente associada com a TAP e negativamente associada com MP e TSP, indicando que altas densidades populacionais estiveram associadas com altas taxas de aparecimento de perfilhos pequenos e baixas taxas de sobrevivência (alta mortalidade) ou vice-versa. Importante comentar que, na ACP, não é levado em consideração o efeito isolado de blocos, tratamentos ou tempo.

No segundo CP, que explicou 29,2\% na variação dos dados, houve associação positiva entre MP, IAF e R e negativa destes com FC. Essa combinação de variáveis indica que perfilhos pequenos estão associados com baixos valores de IAF e de R e elevados valores de FC e vice-versa, ou seja, basicamente evidencia a diferença nas duas formas de se avaliar a relação entre os componentes folha e colmo do pasto. As razões para essa discrepância são discutidas por SACKVILLE HAMILTON et al. (1995) e SBRISSIA\& DA SILVA (2008). Pode parecer paradoxal, mas, no terceiro $\mathrm{CP}$, foi detectada uma associação positiva entre R e FC e negativa destes com a TAP. Esse efeito esteve associado basicamente aos efeitos de época, uma vez que, quando o aparecimento de novos perfilhos foi pequeno, aumentos sazonais em $\mathrm{R}$ implicaram aumentos na relação folha: colmo. O fato indica que, em função da pequena variação registrada em comprimento dos colmos ao longo do ano, o principal mecanismo para o ajuste na forma do perfilho entre as estações esteve relacionado com a capacidade que as plantas têm para se adaptarem às condições de ambiente por meio de aumentos ou reduções em sua área foliar. Resultados semelhantes foram encontrados e discutidos por SBRISSIA \& DA SILVA(2008), usando técnicas de análise univariadas.

Na segunda análise, o objetivo foi o de verificar, principalmente, se a duração de vida das folhas poderia estar associada a um mecanismo de economia de recursos, conforme relatado para outras espécies de plantas (ECKSTEIN et al., 1999). Para tanto, as seguintes variáveis foram utilizadas: densidade populacional de perfilhos (DPP), longevidade de folhas (LF), área foliar específica (AFE), taxa de alongamento de folhas (TAlF) e taxa de senescência foliar (TSF). Nessa análise, ficou evidenciada, no primeiro CP (54,7\% da variação total), a relação inversa entre LF e DPP, AFE e TAlF.

A relação inversa entre essas características poderia estar relacionada com a estratégia de conservação de nutrientes pela planta em épocas de 
Tabela 1 - Análise de componentes principais: eigenvalue, proporção da variância explicada por cada componente, acumulada e correlações entre os componentes e as variáveis originais.

\begin{tabular}{|c|c|c|c|c|c|c|c|}
\hline Eigenvalue & 2,732 & 2,041 & 1,162 & 0,436 & 0,393 & 0,180 & 0,055 \\
\hline Proporção & 0,390 & 0,292 & 0,166 & 0,062 & 0,056 & 0,026 & 0,008 \\
\hline Acumulado & 0,390 & 0,682 & 0,848 & 0,910 & 0,966 & 0,992 & 1,000 \\
\hline Variável $^{*}$ & $\mathrm{CP}_{1}$ & $\mathrm{CP}_{2}$ & $\mathrm{CP}_{3}$ & $\mathrm{CP}_{4}$ & $\mathrm{CP}_{5}$ & $\mathrm{CP}_{6}$ & $\mathrm{CP}_{7}$ \\
\hline DPP & $-0,545$ & $-0,004$ & 0,023 & $-0,407$ & $-0,499$ & 0,104 & 0,526 \\
\hline MP & 0,497 & $-0,315$ & $-0,176$ & 0,058 & 0,020 & 0,641 & 0,457 \\
\hline IAF & $-0,108$ & $-0,645$ & 0,184 & $-0,161$ & $-0,305$ & 0,263 & $-0,592$ \\
\hline $\mathrm{R}$ & 0,022 & $-0,518$ & 0,572 & 0,075 & 0,274 & $-0,410$ & 0,394 \\
\hline FC & $-0,204$ & 0,365 & 0,669 & 0,326 & 0,052 & 0,517 & $-0,038$ \\
\hline TAP & $-0,408$ & $-0,254$ & $-0,336$ & 0,794 & $-0,132$ & $-0,029$ & 0,086 \\
\hline TSP & 0,486 & 0,137 & 0,219 & 0,249 & $-0,750$ & $-0,269$ & 0,029 \\
\hline Eigenvalue & 2,737 & 1,484 & 0,375 & 0,244 & 0,158 & & \\
\hline Proporção & 0,547 & 0,297 & 0,075 & 0,049 & 0,032 & & \\
\hline Acumulado & 0,547 & 0,844 & 0,919 & 0,968 & 1,000 & & \\
\hline DPP & $-0,447$ & 0,413 & $-0,604$ & $-0,490$ & 0,156 & & \\
\hline $\mathrm{LF}$ & 0,558 & 0,070 & $-0,022$ & $-0,603$ & $-0,565$ & & \\
\hline AFE & $-0,523$ & $-0,096$ & 0,697 & $-0,479$ & $-0,045$ & & \\
\hline TAlF & $-0,436$ & $-0,497$ & $-0,322$ & 0,160 & $-0,657$ & & \\
\hline TSF & 0,160 & 0,754 & $-0,210$ & $-0,374$ & 0,471 & & \\
\hline
\end{tabular}

* $\mathrm{DPP}=$ densidade populacional de perfilhos, MP=massa por perfilho, IAF=índice de área foliar, R=razão área foliar: volume, FC=relação folha: colmo, TAP=Taxa de aparecimento de perfilhos, TSP=Taxa de sobrevivência de perfilhos, LF=longevidade de folhas, AFE=área foliar específica, TAlF=Taxa de alongamento de folhas e TSF=Taxa de senescência de folhas.

adversidades climáticas, momento em que normalmente ocorre reduzido alongamento de folhas, redução em DPP e em AFE. O inverso estaria relacionado com estratégias de captura de nutrientes nas épocas de maior disponibilidade de fatores de crescimento. Relação inversa entre área foliar específica e longevidade de folhas já foi demonstrada (RYSER \& URBAS, 2000) e normalmente tem-se assumido que folhas crescendo em ambientes pouco férteis são mais longevas (RYSER, 1996). A correlação negativa entre AFE e longevidade de folhas em capim-marandu foi significativa e corrobora os dados obtidos por esses autores. Já no segundo CP (29,7\% da variação total) foi detectada relação inversa entre TAlF e DPP e TSF, indicando, novamente, uma variação sazonal na alocação de recursos, segundo a qual pastos de capimmarandu aumentam as DPP de perfilhos aéreos (axilares) no período de outono/inverno, como forma de compensar a redução na TAlF e assim aumentar a ocupação de sítios e sua área foliar. O terceiro CP (7,5\% da variação total) mostra uma relação inversa entre DPP e AFE, indicando que, com a redução do perfilhamento no período de inverno (SBRISSIA et al., 2010), as plantas tendem novamente a economizar recursos, aumentando sua AFE. Isso poderia garantir maior velocidade de crescimento com o restabelecimento de condições ambientais adequadas. Conclusões semelhantes foram obtidas por SBRISSIA \& DA SILVA(2008) e SBRISSIA et al. (2010), usando análises de variância simples para análise e interpretação dos dados. Os resultados mostram a potencialidade de se usar a análise de componentes principais na interpretação de dados de pesquisa com plantas forrageiras. As conclusões obtidas são semelhantes àquelas obtidas com técnicas univariadas convencionais, com a vantagem de reduzir o número de variáveis globais em alguns poucos componentes principais.

\section{REFERÊNCIAS}

ECKSTEIN, R.L. et al. Leaf lifespan and nutrient resorption as determinants of plant nutrient conservation in temperatearctic regions. New Phytologist. v.143, p.177-189, 1999.

HAIR, J.F. et al. Multivariate data analysis. 5.ed. Englewood Cliffs: Prentice-Hall, 1998. 730p.

JOLLIFFE, I.T. Principal component analysis. New York: Springer-Verlag, 1986. 271p.

Ciência Rural, v.40, n.3, mar, 2010. 
MATTHEW, C. et al. A modified self-thinning equation to describe size/density relationships for defoliated swards. Annals of Botany, v.76, n.6, p.579-587, 1995. Disponível em: <http:/ /aob.oxfordjournals.org/cgi/reprint/76/6/579>. Acesso em: 27 jan. 2010.

NAVE, R.G. et al. Canonical correlations among chemical, physical and morphological characteristics of Xaraés palisadegrass under rotational grazing. Scientia Agricola, v.66, n.2, p.270-275, 2009. Disponível em: <http:// www.scielo.br/pdf/sa/v66n2/18.pdf>. Acesso em: 27 jan. 2010. doi: 10.1590/S0103-90162009000200018.

RYSER, P. The importance of tissue density for growth and life-span of leaves and roots: comparison of five ecologically contrasting grasses. Functional Ecology, v.10, p.717-723, 1996.

RYSER, P.; URBAS, P. Ecological significance of leaf life span among Central European grass species. Oikos, v.91, n.1, p4150, 2000. Disponível em: <http://www.jstor.org/stable/ 3547472>. Acesso em: 27 jan. 2010.
SACKVILLE-HAMILTON, N.R. et al. In defence of the $-3 / 2$ boundary rule: a re-evaluation of self thinning concepts and status. Annals of Botany, v.76, p.569-577, 1995. Disponível em: <http://aob.oxfordjournals.org/cgi/content/abstract/76/6/ 569>. Acesso em: 27 jan. 2010.

SBRISSIA, A.F. et al. Tiller size/density compensation in grazed Tifton 85 bermudagrass swards. Pesquisa Agropecuária Brasileira, v.38, n.12, p.1459-1468, 2003. Disponível em: <http://www.scielo.br/pdf/pab/v38n12/a13v38n12.pdf>. Acesso em: 27 jan. 2010. doi: 10.1590/S1516-35982008000100005.

SBRISSIA, A.F.; Da SILVA, S.C. Compensação tamanho/ densidade populacional de perfilhos em pastos de capimmarandu. Revista Brasileira de Zootecnia, v.37, n.1, p.3547, 2008. Disponível em: <http://www.scielo.br/pdf/rbz/v37n1/ v37n1a05.pdf>. Acesso em: 27 jan. 2010. doi: 10.1590/S151635982008000100005 .

SBRISSIA, A.F. et al. Tillering dynamics in palisadegrass swards continuously stocked by cattle. Plant Ecology, v.206, n.2, p.349-359, 2010. Disponível em: <http://www.springerlink.com/ content/xg3h80050277224m/>. Acesso em: 27 jan. 2010. doi: $10.1007 / \mathrm{s} 11258-009-9647-7$ 